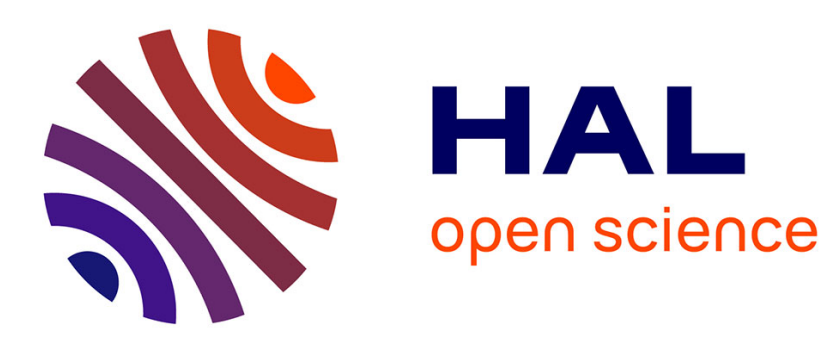

\title{
Si loin, si proches : la difficile association entre circuits courts et commerce équitable
}

\author{
Ronan Le Velly
}

\section{To cite this version:}

Ronan Le Velly. Si loin, si proches: la difficile association entre circuits courts et commerce équitable. Revue Tiers Monde, 2011, 207, pp.133-149. 10.3917/rtm.207.0133 . halshs-00630234

\section{HAL Id: halshs-00630234 \\ https://shs.hal.science/halshs-00630234}

Submitted on 7 Oct 2011

HAL is a multi-disciplinary open access archive for the deposit and dissemination of scientific research documents, whether they are published or not. The documents may come from teaching and research institutions in France or abroad, or from public or private research centers.
L'archive ouverte pluridisciplinaire HAL, est destinée au dépôt et à la diffusion de documents scientifiques de niveau recherche, publiés ou non, émanant des établissements d'enseignement et de recherche français ou étrangers, des laboratoires publics ou privés. 


\title{
Si loin, si proches: la difficile association entre circuits courts et commerce équitable
}

\author{
Ronan Le Velly \\ Montpellier SupAgro \\ UMR Innovation \\ 2 Place Viala. 34060 Montpellier cedex 01. France \\ tel : 04996131 92. fax : 0467545843. \\ levelly@supagro.inra.fr
}

\section{Résumé}

En partant de l'observation de quatre situations de promotion d'un commerce équitable local, l'article décrit les opérations concrètes par lesquelles des acteurs du commerce équitable et des acteurs des circuits courts pensent la convergence entre leurs démarches et engagent des actions communes. L'article montre aussi que ces associations entre commerce équitable et circuits courts s'avèrent difficile à mettre en œuvre, en raison de la différence de projets de développement poursuivis et de l'autonomie relative des mondes du commerce équitable et des circuits courts.

\section{Introduction $^{\bullet}$}

Le commerce équitable, qui avait été initialement pensé comme un outil de développement des producteurs des pays du Sud, est aujourd'hui également envisagé comme un levier pour

\footnotetext{
- Cette recherche a été menée dans le cadre du programme ANR Agriculture et développement durable, projet Collectifs de consommateurs et consommation durable coordonné par Sophie Dubuisson-Quellier.
} 
lutter contre la précarité des agriculteurs des régions du Nord (Chiffoleau, Prevost, 2008). Généralement, les promoteurs d'un «commerce équitable local», «commerce équitable de proximité » ou « commerce équitable Nord-Nord» associent alors à ces termes des formes de circuits courts alimentaires, comme les magasins de producteurs, les marchés fermiers ou les AMAP (Associations pour le Maintien d'une Agriculture Paysanne), qui à leurs yeux permettent d'établir, entre producteurs et consommateurs d'une même région, des relations semblables à celles qui existent dans le commerce équitable.

Une telle association entre circuits courts et commerce équitable n'est pas surprenante au regard des travaux d'économie, de géographie et de sociologie rurale qui identifient ces deux ensembles comme des «systèmes alimentaires alternatifs ». Ainsi, même si leur caractère alternatif mérite d'être considéré avec beaucoup de prudence, tous deux partagent le projet de relations commerciales redéfinies, différentes de celles qui existent dans les circuits «dominants » ou « conventionnels » (Goodman D., 2004 ; Renting et al., 2003 ; Watts et al., 2005). Les valeurs défendues par les circuits courts et le commerce équitable sont alors relativement proches, comme en témoignent les similitudes de vertus que leurs promoteurs respectifs leur associent (équité, autonomisation, authenticité...) et de vices qu'ils attribuent aux circuits auxquels ils s'opposent (exploitation, dépendance, standardisation...) (Hinrichs, 2003 ; Le Velly, 2006). De même, si le commerce équitable diffère de ce que l'on entend habituellement par «circuit court» (Maréchal, 2008), en raison d'une distance géographique et d'un nombre d'intermédiaires important, il s'y affirme aussi une «éthique relationnelle » (Ballet, Carimentrand, 2006), visant l'établissement d'une communauté de destins entre producteurs et consommateurs (Goodman M.K., 2004), qui rappelle les impératifs de proximité et de solidarité fréquemment invoqués par les militants des circuits courts (Lamine, 2005).

L'association entre commerce équitable et circuits courts, exprimée par ces militants d'un « commerce équitable local », mérite néanmoins d'être questionnée. Tout d'abord, à l'image des constats induits par la comparaison entre les expériences de microcrédit des pays du Nord et du Sud (Maystadt, 2004), on peut se demander si les réalités socio-économiques des producteurs bénéficiaires du commerce équitable et des circuits courts ne sont pas trop différentes pour élaborer un argumentaire commun. Ensuite, sans pour autant surestimer la personnalisation des relations entretenues dans les circuits courts (Dubuisson-Quellier, Le Velly, 2008), on peut faire l'hypothèse que l' « éthique relationnelle » est beaucoup plus facile à établir dans les circuits courts que dans le commerce équitable, où la présence des 
intermédiaires s'est considérablement accrue depuis le début des années 1990 (Ballet, Carimentrand, 2006 ; Le Velly, 2006 ; Maldidier, 2010). Plusieurs sociologues, notamment Claire Hinrichs (2003), ont également souligné que les circuits courts étaient parfois mus par un «localisme défensif », une forme de protectionnisme dont on peut se demander si elle est conciliable avec les valeurs de solidarité internationale du commerce équitable. Enfin, dans un contexte où le commerce équitable est de plus en plus fréquemment remis en cause au regard des impacts environnementaux des transports de marchandises, le projet de relocalisation des économies associé aux circuits courts peut apparaître comme lui étant incompatible (Morgan, 2010).

Cet article vise à poursuivre ce questionnement sur le lien entre commerce équitable et circuits courts en s'appuyant sur une enquête de terrain et en adoptant un questionnement inspiré de travaux de sociologie des mouvements sociaux. L'enquête aurait ainsi pu porter, dans une perspective d'économie des filières agroalimentaires et/ou de sociologie des marchés, sur le fonctionnement respectif des circuits courts et des filières du commerce équitable (analyse comparée des chaînes de valeur, des modalités de coordination et de gouvernance...), mais un tel travail aurait sans doute buté sur le constat de la grande hétérogénéité des circuits courts (Maréchal, 2008) comme des filières du commerce équitable (Le Velly, 2006). Pour étudier les distances et les proximités entre ces deux ensembles, je propose plutôt de partir de l'observation de différentes actions menées par quelques acteurs du commerce équitable et quelques acteurs des circuits courts pour promouvoir conjointement un commerce équitable local. L'enjeu se situe alors dans l'observation du travail de construction de lien entre des démarches issues de sous-espaces différents du mouvement social (Mathieu, 2002) et dans la restitution des façons dont ces acteurs pensent la convergence entre leurs projets respectifs et mènent des actions communes.

Quatre situations de promotion d'un commerce équitable local, que je présenterai dans le détail dans la première partie, ont fait l'objet d'une enquête de terrain entre le début de l'année 2005 et le milieu de l'année 2008. Celle-ci a consisté en une analyse qualitative de documents écrits, de nombreuses observations directes et la conduite d'une dizaine d'entretiens exploratoires et de 32 entretiens semi-directifs. ${ }^{1}$ Cette enquête ethnographique ne

\footnotetext{
${ }^{1}$ Les documents étudiés étaient publics (tracts, sites internet...) ou privés (compte-rendus d'assemblées générales, journaux internes...), actuels ou d'archives. Les observations ont été menées sur des points de vente (boutiques, marchés) et lors de conférences et de réunions. Les 32 personnes interviewées, toutes partiesprenantes des cas étudiés, étaient pour moitié issues du commerce équitable ( 7 bénévoles et 2 salariés
} 
peut prétendre à la représentativité. En revanche, les cas étudiés ont été retenus de façon à rendre compte d'actions diverses (de l'ouverture d'une boutique couplant circuits courts et commerce équitable à l'organisation d'une campagne nationale pour défendre l'idée d'un commerce équitable local) impliquant des organisations elles-mêmes hétérogènes (dans leurs tailles, leurs valeurs, leurs modalités d'action...). De même, les entretiens n'ont pas fait l'objet d'une véritable analyse de discours, mais leur contenu a été codé manuellement, afin de restituer les points de vue exprimés le plus finement possible et de faire apparaître leurs récurrences.

Les résultats de cette enquête seront analysés dans les deuxièmes et troisièmes parties. J'identifierai deux logiques qui traversent les quatre situations étudiées, une logique d'association et une logique de séparation. Le sentiment qui ressortira de ce compte-rendu sera alors celui d'une «proximité lointaine» entre commerce équitable et circuits courts : d'un côté, des opérations concrètes construisent des associations entre les démarches et les acteurs des circuits courts et du commerce équitable (partie 2), mais de l'autre, ces associations sont contestées et les coopérations demeurent difficiles (partie 3).

\section{Quatre situations de promotion d'un commerce équitable local}

\section{L'inscription du commerce équitable Nord-Nord à l'agenda de la Fédération Artisans du}

\section{Monde}

En 2004, le conseil d'administration de la Fédération Artisans du Monde (FADM) ${ }^{2}$ a engagé un processus de consultation de ses militants visant à rédiger le Projet associatif d'Artisans du Monde. Cette consultation intervenait dans un contexte de crise, principalement en raison de profonds désaccords concernant la stratégie commerciale de la centrale d'importation du réseau, Solidar'Monde. A l'époque, la FADM ne possédait que $24 \%$ du capital de sa centrale d'importation et la majorité était détenue par le Comité Catholique Contre la Faim et pour le Développement (CCFD). Ce dernier envisageait d'introduire quelques produits de la gamme de Solidar'Monde en grandes surfaces, mais cette proposition était catégoriquement refusée

d'associations locales, 3 salariés d'associations nationales, 5 commerçants professionnels) et pour moitié issues du monde agricole français ( 9 agriculteurs engagés en circuits courts, 4 salariés d'organisations de développement agricole, 2 militants associatifs).

${ }^{2}$ La FADM est le principal mouvement associatif français de commerce équitable. En 2010, elle regroupait 160 groupes locaux et revendiquait 6000 militants. Sur son histoire et son fonctionnement: Le Velly (2006), Maldidier (2010). 
par les administrateurs de la Fédération. La consultation a débouché sur un vote, lors de l'assemblée générale de 2005, par lequel les représentants des groupes Artisans du Monde se sont prononcés pour un refus strict de ce mode de commercialisation et pour un rachat des actions de Solidar'Monde détenues par le CCFD.

Dans le cadre de cette consultation, d'autres questionnements ont été introduits. Les administrateurs de la FADM ont ainsi défendu l'inscription de la promotion d'un commerce équitable avec des producteurs des pays du Nord à l'agenda du mouvement. Ils plaidaient pour que le mouvement s'inspire d'initiatives de commercialisation en circuits courts et se rapproche d'organisations favorables à une « agriculture durable », telles que la Confédération paysanne, la Fédération Nationale de l'Agriculture Biologique et la Fédération Nationale des CIVAM (Centre d'Initiatives et pour Valoriser l'Agriculture et le Milieu rural). ${ }^{3}$ Lors de l'assemblée générale de 2005, cette question a été beaucoup moins débattue que celle de la stratégie commerciale, mais elle a tout de même fait l'objet de l'adoption à la quasi-unanimité d'une motion favorable à la «promotion d'un commerce local équitable et solidaire au Nord ». L'année suivante, une section du Plan d'activité associatif a également été consacrée à ce point et a établi la possibilité pour les boutiques de vendre des produits locaux à concurrence de $10 \%$ de leur chiffre d'affaires. Enfin, un salarié a été embauché pour accompagner les groupes Artisans du Monde dans leurs démarches et la Fédération dans ses réflexions.

Cette orientation a été extrêmement rapide. Pourtant, la question d'un commerce équitable Nord-Nord avait déjà été posée par le passé, mais autant lors de l'assemblée générale de 1988 que de celle de 1995, il avait été avancé qu'un tel positionnement risquerait de détourner le mouvement de sa mission première de soutien aux producteurs du Tiers-Monde et aucune suite favorable n'avait été donnée. La thématique du commerce équitable Nord-Nord n'est donc peut-être pas aussi évidente que ne le laisse supposer la quasi-unanimité qui a présidé à son inscription au Projet associatif d'Artisans du Monde.

\section{La campagne «Pour un commerce équitable partout! Changeons la loi!»}

«Pour un commerce équitable partout! Changeons la loi !» est le nom d'une campagne nationale lancée en 2006 par Breizh Ha Reizh, Minga et la Confédération paysanne dans le but de contester l'article 60 de la loi sur les PME du 2 août 2005 définissant le commerce

\footnotetext{
${ }^{3}$ Pour une présentation respectivement des circuits courts et de l'«agriculture durable »: Maréchal (2008), Deléage (2005).
} 
équitable comme des «échanges de biens et de services entre des pays développés et des producteurs désavantagés situés dans des pays en développement » (je souligne).

Minga et Breizh Ha Reizh sont deux collectifs d'acteurs du commerce équitable, revendiquant en 2008 respectivement 70 et 25 organisations adhérentes (généralement de petites structures commerciales, importateurs et/ou détaillants spécialisés dans le commerce équitable), réparties sur le territoire national pour le premier, en Bretagne pour le second. Minga et Breizh Ha Reizh défendent une conception du commerce équitable en rupture avec la stratégie de commercialisation en grandes surfaces de Max Havelaar France, on y reviendra, mais sont également critiques à l'égard de la présence de bénévoles dans le réseau Artisans du Monde, qu'ils perçoivent comme une source de concurrence déloyale et comme une entrave à l'établissement d'un commerce réellement équitable d'un bout à l'autre de la filière. Enfin, une dernière spécificité de Minga et de Breizh Ha Reizh est d'avoir été parmi les premiers à clairement plaider pour une définition du commerce équitable incluant des relations NordNord.

En amont de la campagne «Pour un commerce équitable partout!», Minga et Breizh Ha Reizh avaient ainsi participé à plusieurs réunions d'une commission de l'Agence Française de Normalisation (AFNOR) dédiée au commerce équitable. Cette commission avait débouché au printemps 2005 sur la publication d'un «fascicule de documentation» dans lequel les représentants de Minga et de Breizh Ha Reizh avaient obtenu que des relations établies avec des producteurs locaux puissent se revendiquer du commerce équitable si leurs situations économiques le justifiaient. Cependant, notamment sous la pression de Max Havelaar France et de la FADM qui voyaient dans d'autres passages du texte une définition « au rabais » du commerce équitable, le fascicule n'avait ensuite pas été validé. Un rapport parlementaire avait alors été commandé par le Premier ministre Jean-Pierre Raffarin au député du Bas-Rhin Antoine Herth et, quelques semaines plus tard, un amendement sur le commerce équitable était voté dans le cadre de la loi sur les PME. Ce texte est court et laisse en suspens beaucoup de questions mais, contrairement à celui du fascicule de l'AFNOR, il propose une définition qui est fermée aux relations Nord-Nord.

La campagne «Pour un commerce équitable partout! Changeons la loi » a été initiée à la suite de ces épisodes. Au moment de son lancement, Minga et Breizh Ha Reizh ont obtenu que la Confédération paysanne cosigne l'appel. Cet engagement a été vécu comme une opportunité d'ancrer les revendications de la campagne au-delà de questions propres au commerce équitable et d'en assurer la résonance dans le monde agricole français. Pour autant, 
sur ces deux points, la campagne a plutôt échoué. La Confédération paysanne ne s'est guère impliquée et a très peu relayé l'information auprès de ses adhérents et sympathisants.

\section{Des actions en faveur d'un commerce équitable local en Bretagne}

Deux initiatives, menées en Bretagne à partir de 2004-2005, peuvent également être détaillées. La première a été portée par Ingalan, une association de promotion du commerce équitable dont le fondateur, Hervé Legal, a par ailleurs eu un rôle central dans la création de Breizh Ha Reizh et a représenté cette dernière lors des négociations de l'AFNOR. En 2005, Ingalan a initié un collectif nommé «Agir en Bretagne pour développer le commerce équitable local et international, moteur d'une économie sociale et solidaire », qui a réuni des acteurs du commerce équitable, par ailleurs membres de Breizh Ha Reizh, et trois organisations du monde agricole breton, la Confédération paysanne du Morbihan, le Groupement d'Agriculture Biologique du Morbihan et la Fédération des CIVAM de Bretagne. Des discussions ont alors été engagées pour l'élaboration d'une «Charte du commerce équitable local en Bretagne » et la création d'un identifiant qui permettrait de distinguer les circuits de commercialisation et les produits se référant à cette démarche. La même année, Ingalan a fait appel à un élève ingénieur agronome pour un stage de fin d'étude consistant à repérer une première série d'initiatives allant dans ce sens. Ce travail a permis la rédaction d'un tract identifiant sur la Bretagne une dizaine de démarches de commercialisation en circuits courts qualifiées de « commerce équitable local».

A la même époque, la Coordination des Associations de Solidarité Internationale (CASI) de Bretagne éditait un guide «des acteurs et des pratiques du commerce équitable en Bretagne » recensant 53 organisations et distinguant les démarches relevant d'un commerce équitable Nord-Sud, celles mêlant Nord-Nord et Nord-Sud, et celles s'inscrivant exclusivement dans le Nord-Nord. Dans ce dernier cadre, le guide citait Bro An Are, un magasin tenu par une dizaine d'agriculteurs des Monts d'Arrée. Le fait qu'un tel guide, édité par un collectif d'associations de solidarité internationale, fasse référence à un magasin fermier mérite une attention particulière. Un élément important est qu'à l'époque, Gilles Maréchal, le président de la CASI était un militant d'une association de solidarité avec le Brésil mais aussi, à titre professionnel, coordinateur de la Fédération des CIVAM de Bretagne. Il était donc un interlocuteur particulièrement capable de porter la thématique du commerce équitable NordNord auprès des acteurs de la solidarité internationale, mais aussi auprès des agriculteurs engagés en circuits courts dont les CIVAM soutiennent les démarches. 
Ces situations illustrent la possibilité d'établir autour de l'idée de commerce équitable local des relations entre les acteurs du commerce équitable et des circuits courts. Pour autant, les difficultés rencontrées ne doivent pas être négligées. Ainsi, le collectif mis en place par Ingalan a rapidement cessé de se réunir et n'est pas parvenu à produire la charte et l'identifiant pour lequel il avait été constitué. La CASI de Bretagne ne s'est pas non plus imposée comme l'instance motrice de collaborations entre acteurs du commerce équitable et du monde agricole. A partir de 2006, elle est même devenue le lieu de vifs débats internes aux militants bretons du commerce équitable, opposant des membres de Breizh Ha Reizh et de Max Havelaar Bretagne, les premiers défendant une définition ouverte du commerce équitable, les seconds entendant accorder la priorité aux producteurs des pays du Sud. Un paradoxe de cette situation est que les agriculteurs bretons directement concernés par les questions posées n'ont pas pris part aux débats. Les discussions se sont plus refermées sur des enjeux internes au monde du commerce équitable qu'elles ne se sont ouvertes vers l'extérieur.

\section{Différentes formes de collaborations entre militants d'Artisans du Monde et agriculteurs} locaux

On a vu que la FADM a inscrit à son agenda la promotion d'un commerce équitable NordNord, mais qu'en est-il au niveau des associations locales? Un premier constat est que la plupart d'entre elles entretiennent des collaborations avec des agriculteurs locaux, mais que ces collaborations demeurent relativement distantes. Par exemple, il est fréquent que des groupes Artisans du Monde tiennent un stand lors de manifestations organisées par des organisations paysannes, revendent un ou deux produits d'un agriculteur local ou servent de lieu de dépôt pour une AMAP. Toutes ces actions ne font pas l'objet de débats mais, de façon symptomatique, elles tendent à s'arrêter aux premières tracasseries d'ordre pratique (comptabilité, livraison) ou dès que le bénévole qui en a la charge cesse de s'en occuper. Quelques groupes Artisans du Monde ont cependant développé des collaborations avancées avec des collectifs d'agriculteurs. Deux cas riches d'enseignements méritent d'être exposés.

La Halte fermière est un magasin fermier qui a ouvert ses portes en 2005 à Voiron, en région grenobloise, suite au travail de neuf agriculteurs et d'une bénévole du groupe Artisans du Monde de la ville, par ailleurs salariée de la Chambre d'agriculture de l'Isère. Au moment où je m'y suis rendu, en 2007, le magasin proposait une large gamme de produits alimentaires issus du commerce équitable, trois membres d'Artisans du Monde participaient à tour de rôle aux permanences de vente et un d'entre eux prenait part à titre consultatif au conseil d'administration. Ce partenariat était donc avancé, mais il ne se faisait pas sans heurts. Malgré 
deux années de collaboration, une partie des neuf agriculteurs ne trouvait toujours pas de points communs entre leur démarche et celle du commerce équitable. Ils acceptaient la présence des produits des pays du Sud comme un utile complément de gamme, mais se sentaient très mal à l'aise pour en parler et refusaient formellement que le magasin communique en ne distinguant pas clairement les démarches fermière et équitable.

Dans le centre-ville de Grenoble, la boutique Soli'gren a été initiée en 2006, dans le cadre d'un projet impliquant de nombreux partenaires, au premier rang desquels le groupe local d'Artisans du Monde. Cette boutique vend des produits du commerce équitable et des produits alimentaires achetés auprès de producteurs locaux. Pour autant, au moment de mon enquête, les rapports entre militants du commerce équitable et agriculteurs locaux étaient très ténus. Les premiers ne se sentaient pas capables de vendre les produits des seconds et les seconds ne participaient pas à la vie de la boutique (ni aux réunions, ni aux permanences, certains n'assurant même pas la livraison de leur marchandise). Il se posait également la question de la concurrence entre produits issus du commerce équitable et produits locaux. Dans le cadre de magasins fermiers (comme la Halte fermière), les produits issus du commerce équitable qui viendraient concurrencer les produits locaux (miel, vin, confitures, jus de fruits), ne sont généralement pas proposés. A Soli'gren, le choix a été fait de conserver les deux gammes, quitte à exposer sur une même étagère une confiture de l'Isère vendue $50 \%$ plus chère qu'une confiture du Laos. Certes, comme me l'ont expliqué les responsables de Soli'gren, cette situation invite à réfléchir à la mondialisation économique et la décomposition des prix. Mais, on comprend aussi que les producteurs locaux qui voient leurs produits ainsi concurrencés n'acceptent pas forcément cette invitation avec le sourire.

\section{Construire les associations entre commerce équitable et circuits courts}

L'observation de ces quatre situations suggère que la mise en relation entre le commerce équitable et les circuits courts, pour la reconnaissance de l'idée de commerce équitable local ou la construction de circuits de distribution communs, ne se fait pas «toute seule». Non seulement elle suppose un travail spécifique de construction d'associations, que je vais maintenant analyser, mais de surcroît ce travail bute sur certaines difficultés, sur lesquelles je reviendrai dans la troisième partie. Pour décrire ces éléments et gagner en généralité, je mobiliserai des références théoriques qui sont aujourd'hui fréquemment utilisées de concert dans les travaux de sociologie des mouvements sociaux cherchant à décrire les conditions de 
réalisation d'actions communes entre organisations issues de sous-espaces différents du mouvement social (Mathieu, 2002 ; McAdam et al., 2001).

\section{Des opérations visant à établir une connexion entre les cadres interprétatifs du commerce} équitable et des circuits courts

La sociologie des mouvements sociaux parle de connexion de cadres ( frame bridging ») pour décrire les opérations par lesquelles un lien est établi entre les cadres interprétatifs de deux groupes défendant des causes jusqu'ici distinctes. Une telle entrée, même si elle ne suffit pas à rendre compte de l'ensemble des conditions de l'action collective et demande à être complétée par la suite de l'analyse (Mathieu, 2002), est particulièrement utile pour décrire le travail d'association mené par les partisans d'un commerce équitable local : l'association entre commerce équitable et circuits courts passe alors par la connexion entre leurs «cadres d'injustice » (Gamson, 1995) et « cadres de diagnostic » (Benford, Snow, 2001) respectifs.

Même si les promoteurs du commerce équitable Nord-Nord ont conscience que les situations des producteurs marginalisés des pays du Nord et du Sud ne sont pas les mêmes, ils énoncent qu'elles sont tout aussi dramatiques. Reprenant le discours qu'il tient généralement en public, un militant de Breizh Ha Reizh expliquait : «Je refuse l'échelle de la misère. (...) C'est vrai que les conditions de vie sont plus dures là-bas. Mais ici un producteur qui est obligé de mettre la clé de son exploitation sous la porte, après trois, quatre ou cinq générations, et qui se tire une balle dans la tête derrière... Je suis glauque, mais on parle souvent du taux de suicide dans le Trégor, dans les Pays de Loire... Elle est où la différence de la misère ici ?» (T., entretien, 27 mars 2006). La connexion de cadres se poursuit par l'identification de causalités et de responsables communs à la situation des producteurs marginalisés du Nord et du Sud. L'accusation vise alors généralement la logique de la mondialisation libérale et les firmes multinationales qui l'incarnent. Par exemple, dans une des contributions écrites des groupes locaux Artisans du Monde envoyées en vue de la rédaction du Projet associatif, il était écrit : «Comment dissocier les conditions économiques des pays du Sud et celles du Nord tant elles sont liées? Exploitation au Sud, délocalisations au Nord sont bien issues des mêmes recherches de profit au mépris de toute considération humaine. » La proximité est enfin montrée au regard des solutions à adopter. Au moment de la rédaction du Projet associatif, les administrateurs de la Fédération ont tout particulièrement insisté sur le fait que les promoteurs des circuits courts partagent avec le mouvement Artisans du Monde le souhait de sortir des circuits de la grande distribution. Plusieurs principes du commerce équitable trouvent également des équivalences relativement spontanées dans les circuits courts, qu'il s'agisse de 
l'établissement d'une relation «directe », du travail avec des «petits producteurs », du paiement d'un «prix juste » ou de la « connaissance des producteurs derrière les produits ».

2. Des personnes et des objets à l'interface entre acteurs du commerce équitable et acteurs des circuits courts

L'association entre commerce équitable et circuits courts peut également être retracée au regard des relations entre acteurs de ces deux ensembles. Sur ce point, les situations observées confirment le rôle des «brokers» (courtiers) agissant à l'interface entre sous-espaces du mouvement social (McAdam et al., 2001). Le cas le plus exemplaire que l'on a exposé est sans doute celui de Gilles Maréchal, président de la Coordination des Associations de Solidarité Internationale (CASI) de Bretagne, par ailleurs coordinateur de la Fédération régionale des CIVAM. En 2004, lorsqu'il initie la rédaction du Guide des acteurs et des pratiques du commerce équitable en Bretagne, les cercles bretons du commerce équitable et des circuits courts sont relativement disjoints et le guide de la CASI n'aurait en son absence vraisemblablement pas évoqué les circuits courts. D'autres brokers ont également émaillé l'exposé de la première partie. Par exemple, lorsque Hervé Legal, le fondateur d'Ingalan se tourne vers un élève ingénieur agronome pour identifier les expériences de vente en circuits courts qui pourraient être qualifiées de «commerce équitable local», il le fait parce qu'il connaît lui-même mal le monde agricole et qu'il a besoin de s'appuyer sur une personne qui possède cette compétence. On peut enfin se remémorer la bénévole d'Artisans du Monde Voiron, par ailleurs salariée de la Chambre d'agriculture de l'Isère, qui est intervenue dans l'ouverture la Halte fermière.

Outre le rôle des personnes, on peut souligner le rôle des objets dans la construction des relations entre acteurs et dans les «traductions » associant les problèmes et les solutions identifiés dans leurs démarches respectives (Callon, 1988). Projet associatif d'Artisans du Monde, «Fascicule de documentation» de l'AFNOR, tract d'Ingalan identifiant les démarches de commerce équitable local, guide des acteurs bretons du commerce équitable Nord-Nord/Nord-Sud, magasins et étagères où sont vendus côte à côte des produits locaux et des produits issus du commerce équitable : tous ces objets peuvent être décrits comme des objets intermédiaires, au sens de Dominique Vinck (1999), c'est à dire des objets qui marquent la réalisation d'une étape dans les associations, stabilisant les relations établies et permettant d'envisager d'en produire de nouvelles. La « Charte du commerce équitable local en Bretagne », telle qu'elle avait été envisagée par Ingalan aurait également agi de la sorte. Elle aurait matérialisé le travail de coopération mené entre les acteurs du commerce équitable 
et des circuits courts et permis d'envisager ensuite la création d'un logo identifiant les produits ou les organisations s'y référant. Cette charte illustre en outre l'intérêt d'observer ce que Susan Star et James Griesemer (1989) nomment des objets-frontière, des objets ayant des significations suffisamment ambivalentes pour faire sens auprès de différents acteurs, mais suffisamment robustes pour permettre leur coopération.

III. Des associations contrariées par l'autonomie du commerce équitable et des circuits courts

\section{Deux démarches qui ne renvoient pas aux mêmes projets de développement}

Un vecteur important de l'association entre commerce équitable et circuits courts consiste dans la mise en équivalence, en dépit de niveaux de développement inégaux, de la situation également dramatique des producteurs marginalisés des pays du Sud et du Nord. Or, cette connexion est peu en phase avec le ressenti de beaucoup de militants du commerce équitable, particulièrement d'Artisans du Monde, et de la plupart des agriculteurs engagés en circuits courts. Même s'ils connaissent les difficultés de nombre d'agriculteurs de nos régions, même s'ils comprennent les raisonnements qui concluent à la convergence entre commerce équitable et circuits courts, il existe à leurs yeux une trop grande différence entre le niveau de développement des pays du Nord et du Sud pour complètement penser l'équivalence entre commerce équitable et circuits courts. Ainsi, les agriculteurs que j'ai rencontrés étaient généralement peu à l'aise avec l'idée qu'on qualifie leur vente directe de «commerce équitable », d'autant qu'une bonne partie d'entre eux ne se sentaient pas en situation de précarité. De même, un militant d'Artisans du Monde Voiron à qui j'avançais que de nombreux agriculteurs français ne touchent pas plus que le RMI me retournait ainsi : «Oui bien sur, ils sont RMIstes, ils touchent quand même quelque chose. Au sud, ils touchent quoi ? Non c'est sans comparaison. » (M., entretien, 15 mars 2007)

Cette façon de juger incomparables les situations du Sud et du Nord fait que les projets de développement du commerce équitable et des circuits courts sont aussi vécus de façons distinctes. Les agriculteurs que j'ai rencontrés, lorsqu'ils parlent des produits du commerce équitable qu'ils proposent en plus des leurs dans leurs points de vente, disent ainsi vouloir «aider » ou « rendre service » aux producteurs du Tiers-Monde, traduisant le sentiment d'une

relation non-égalitaire, plus tournées vers le caritatif que vers le commercial. D'ailleurs, même si les militants du commerce équitable définissent leur action en opposition aux 
logiques de charité (Le Velly, 2006), le constat a été fréquemment effectué que ces logiques persistent tout de même dans le rapport qu'ils entretiennent avec les « petits producteurs du Sud» dont ils idéalisent même parfois la pauvreté (Lemay, 2006). A l'inverse, les circuits courts vont être défendus, notamment en milieu rural, non pas comme une façon de soutenir les producteurs, mais de rouvrir des commerces de proximité, de créer de la valeur ajoutée sur place, d'embaucher ou d'installer des jeunes de la région, etc. Le consommateur des circuits courts n'est alors pas pensé comme effectuant une bonne action en faveur de producteurs défavorisés au loin, mais comme agissant en faveur d'un développement local dont au final il bénéficie.

Dans ce dernier cas, la démarche poursuivie par les circuits courts correspond bien au projet d'une « économie morale », au sens précis où l'entendait Edward Thompson (1993), c'est à dire une économie où les échanges commerciaux ne lèseraient pas les participants de l'échange et ne nuiraient pas à la survie ou au développement de la communauté à laquelle ils appartiennent. Mike Goodman (2004) a particulièrement bien décrit comment ces valeurs d'économie morale peuvent être présentes dans les circuits du commerce équitable. Par la transmission d'informations sur l'histoire des groupements de producteurs, la reprise de leurs témoignages, l'affichage de leurs photos ou l'organisation de leur visite, les promoteurs du commerce équitable essaient de générer un sentiment de communauté d'appartenance entre producteurs du Sud et consommateurs du Nord. Pour autant, le travail de comparaison effectué dans les paragraphes précédents suggère qu'un tel sentiment est plus artificiel dans le cadre du commerce équitable et plus immédiat dans un contexte où producteurs et consommateurs partagent effectivement une appartenance communautaire.

\section{Deux mondes qui ne partagent pas les mêmes enjeux et se connaissent mal}

La section précédente montre que les démarches du commerce équitable et des circuits courts ne sont pas vraiment pensées comme relevant d'une même logique, mais une telle référence ne suffit pas à expliquer les difficultés de l'action collective (Mathieu, 2002). En inscrivant mon analyse dans le constat classique d'une différenciation de l'espace social en sousensembles relativement autonomes, structurés autour de rapports d'interdépendance et d'enjeux spécifiques (Bourdieu, 1984), je vais maintenant souligner la distance existant entre le monde du commerce équitable et celui des circuits courts.

Dans cette perspective, les difficultés de collaborations peuvent être expliquées par des priorités différentes, associées à des socialisations militantes différentes. Les situations de 
concurrence entre produits locaux et produits issus du commerce équitable sont à cet égard révélatrices. Ainsi, pour les promoteurs des circuits courts, l'importation même équitable de produits pouvant être achetés localement sera plutôt considérée avec suspicion, d'autant qu'elle apparaît comme écologiquement contestable. Cette position est compréhensible pour les militants du commerce équitable, mais ils n'iraient généralement pas jusqu'à exclure le miel du Chiapas ou les confitures du Laos de leurs propres boutiques, même s'ils y vendent aussi des produits locaux.

De même, il faut expliquer que lorsque la FADM, Breizh Ha Reizh ou Minga promeuvent l'établissement d'un commerce équitable Nord-Nord, ils contestent dans le même mouvement la stratégie de Max Havelaar France de commercialisation en grandes surfaces. Les deux controverses sont liées. Dès lors que les travailleurs marginalisés du Nord sont pensés comme bénéficiaires du commerce équitable, le sort des employés et des fournisseurs de la grande distribution ne peut être ignoré et la stratégie de vente en grandes surfaces est «incohérente », «hypocrite» ou «contradictoire», pour reprendre des termes qui ont accompagné les discussions préalables à la rédaction du Projet associatif d'Artisans du Monde. La promotion du commerce équitable Nord-Nord peut alors être analysée comme une stratégie visant à imposer une nouvelle définition des enjeux du monde du commerce équitable, pour contester le leadership symbolique de son acteur dominant et peut-être en retirer un gain financier, puisque toutes ces organisations sont aussi en concurrence pour capter les financements liés à la promotion « du » commerce équitable. ${ }^{4}$

Ce rapport à Max Havelaar est essentiel pour tout acteur du commerce équitable, mais, transposé dans le monde des circuits courts, il fait beaucoup moins sens. Du fait de leur absence de fréquentation durable du monde du commerce équitable, les acteurs des circuits courts ne savent pas forcément comment se positionner face aux arguments contradictoires qu'ils entendent de part et d'autre au sujet de la vente en grande distribution des produits du commerce équitable. Surtout, beaucoup d'entre eux ne souhaitent pas prendre parti parce qu'ils n'ont pas d'intérêt pour le faire (Bourdieu, 1994). Aussi bien les salariés des CIVAM de Bretagne qui avaient participé aux réunions organisées par Ingalan en vue de la rédaction d'une «Charte du commerce équitable en Bretagne» que les responsables de la Confédération paysanne qui avaient initialement soutenu l'appel «Pour un commerce équitable partout! » m'ont expliqué qu'ils avaient cessé de s'y impliquer dès lors qu'ils avaient eu l'impression que ces projets visaient autant à contester l'action de Max Havelaar

\footnotetext{
${ }^{4}$ Pour un raisonnement similaire : AGRIKOLIANSKY et al., 2005.
} 
que de soutenir les agriculteurs locaux ou l'agriculture paysanne. Ce point est également parfaitement exprimé dans le témoignage d'un agriculteur de la région rennaise, membre d'un magasin fermier et administrateur de la Fédération des CIVAM de Bretagne :

«On voudrait nous impliquer dans une bagarre pour laquelle on n'est au courant de rien. En allant au colloque organisé par Breizh Ha Reizh, je me suis rendu compte que les gens se bagarraient, parce que certains revendiquaient le Nord-Sud et que d'autres disaient "non, non c'est le Nord-Nord" et traitaient les premiers de néo-colonialistes... (...) Finalement on s'est dit que ça serait bien de faire un rayon de commerce équitable dans notre magasin et c'est là que les ennuis ont commencé. Parce qu'on a commencé à se poser des questions. On se rend compte que c'est la foire d'empoigne dans le système. (...) D'une réunion à l'autre, il nous vient des informations différentes. On ne sait plus du tout. Des fois, on se demande pourquoi on s'ennuie avec ça. »(J., entretien, 6 mars 2006)

L'existence de deux mondes sociaux relativement distincts implique enfin, même si cela n'est pas systématique (Gateau, 2007), que la plupart des acteurs se connaissent mal et ne se font pas forcément confiance. Les agriculteurs engagés en vente directe, intéressés par la vente de produits du commerce équitable, sont ainsi souvent désemparés devant la multiplication des marques et labels. Symétriquement, si l'agriculture biologique évoque généralement des connotations positives aux acteurs du commerce équitable, que penser de l'agriculture durable, de l'agriculture raisonnée, de l'agriculture paysanne ou de l'agriculture fermière ? Dans le magasin Soli'gren, les bénévoles d'Artisans du Monde vendent des produits, provenant d'agriculteurs membres de l'ADAYG (Association pour le Développement de l'Agriculture dans l'Y Grenoblois), une organisation de promotion des circuits courts. Mais, outre cette origine géographique, ils n'en connaissent pas grand chose. La charte de l'ADAYG est certes disponible dans le magasin, mais son contenu demeure obscur pour une personne extérieure au monde agricole. Cela contribue à la faible motivation des militants d'Artisans du Monde à mettre en valeur les produits locaux et à expliquer aux clients la démarche Nord-Nord du magasin.

La méconnaissance peut même se coupler avec de la méfiance. Lorsque la Halte fermière a ouvert ses portes à Voiron, une partie des producteurs locaux adhérents étaient hostiles aux permanences des militants d'Artisans du Monde dans la boutique et leur ont régulièrement manifesté qu'ils «n'étaient pas du même monde ». Ils leur reprochaient notamment leur totale ignorance de la vie agricole, ignorance qui pouvait effectivement transparaître lorsque 
certains d'entre eux critiquaient le manque de régularité des approvisionnements sans avoir conscience des rythmes imposés par la production fermière. De même, il n'a jamais été possible de relayer à la Halte fermière les campagnes de plaidoyer menées par la FADM, notamment celles mettant en cause les politiques agricoles et commerciales européennes. Il faut dire que les raccourcis de certains slogans comme «Exportation de poulets. L'Europe plume l'Afrique » auraient pu laisser entendre que l'ensemble des agriculteurs européens s'enrichit aux dépens des paysans africains.

\section{Conclusion}

Au sujet des incompréhensions qui émaillent les premières années d'ouverture de la Halte fermière et de Soli'gren, une animatrice de l'ADAYG, très favorable à l'idée de commerce équitable local, me mettait en garde quant au message que je comptais transmettre dans mon compte-rendu. Selon elle, c'est précisément par le biais d'expériences comme celles-ci qu'il est possible d'établir un dialogue et de penser des problématiques communes au monde du commerce équitable et au monde agricole. Je souscris intégralement à cette remarque, qui place sur le devant de l'argumentation les logiques d'association, mais pense qu'il est également important d'avoir conscience des logiques de séparation qui constituent l'autre face des processus en cours. Il est vrai que l'organisation de mon exposé, présentant d'abord les opérations d'association puis les tendances à la séparation, tend à mettre l'accent plus sur le second que sur le premier point. Cela ne signifie pas que les collaborations envisagées entre les acteurs du monde agricole et des circuits courts soient vouées à l'échec. Ce choix d'exposition résulte du caractère problématique des démarches que j'ai observées et l'étude d'autres situations aurait peut-être suggéré une autre tonalité. De même, si dans les années à venir les collaborations sont plus abouties, les comptes-rendus gagneront à partir de la situation d'autonomie relative des deux mondes pour aller vers la description des associations menées avec succès. Pour autant, gageons que même dans ce cas, la tension entre les deux logiques décrites sera toujours observable. Par exemple, dans un article consacré à l'engagement de la ville de Bristol dans le programme Fairtrade City, il est surtout mis l'accent sur la convergence entre les démarches de commerce équitable et de circuits courts, mais les auteurs évoquent aussi des tensions semblables à celles que j'ai décrites (Malpass et al., 2007). En somme, en repensant à la notion d'objet-frontière, il me semble que les coopérations entre acteurs du commerce équitable et des circuits courts autour du commerce équitable local gagnent à être pensées comme «partagées » au double sens de «communes» 
et de «divisées » (Tornatore, 2000). L'ambiguïté d'une notion comme celle de commerce équitable local est alors à la fois ce qui permet le rapprochement, mais aussi ce qui complique l'accomplissement d'une action collective approfondie.

\section{Bibliographie}

Agrikoliansky E., Filleule O., Mayer N., 2005, «Introduction. Aux origines de l'altermondialisme français » in Agrikoliansky E., Filleule O., Mayer N. (dir.) L'altermondialisme en France. La longue histoire d'une nouvelle cause, Paris, Flammarion, pp. 13-42.

Ballet J., Carimentrand A., 2006, «Le commerce équitable: des labels à l'éthique relationnelle » in Chiffoleau Y., Dreyfus F., Touzard J.M. (dir.) Les nouvelles figures des marchés agroalimentaires, Versailles, Quae éditions, pp. 61-71.

Benford R., Snow D., 2001, «Framing processes and social movements: an overview and assessment », Annual review of sociology, $\mathrm{n}^{\circ} 26$, pp. 611-639.

Bourdieu P., 1984, Questions de sociologie, Paris, Editions de Minuit.

Bourdieu P., 1994, Raisons pratiques. Sur la théorie de l'action, Paris, Seuil.

Callon M. (dir.), 1988, La science et ses réseaux. Genèse et circulation des faits scientifiques, Paris, La Découverte.

Chiffoleau Y., Prevost B., 2008, «Consommer local. Plus qu'une mode, une éthique ?», Courrier de la planète, $\mathrm{n}^{\circ} 87$, pp. 48-52.

Deléage E., 2005, «L'agriculture durable : utopie ou nécessité ? », Mouvements, ${ }^{\circ}$ 41, pp. 64-69.

Dubuisson-Quellier S., Le Velly R., 2008, «Les circuits courts, entre alternative et hybridation », in Maréchal G. (dir.) Les circuits courts alimentaires, Dijon, Editions Educagri, pp. 105-112.

Gamson W., 1995, Talking politics, Cambridge, Cambridge University Press.

Gateau M., 2007, « Du bouche-à-oreille à l'entretien d'embauche. Le recrutement dans les associations de commerce équitable », Sociologies pratiques, $\mathrm{n}^{\circ} 15, \mathrm{pp} .123-134$.

Goodman D., 2004, « Rural Europe redux? Reflections on alternative agro-food networks and paradigm change », Sociologia ruralis, vol. 44, n 1 , pp. 3-14. 
Goodman M.K., 2004, «Reading fair trade: political ecology, imaginary and the moral economy of fair trade foods », Political geography, vol. 23, n 7, pp. 891-915.

Hinrichs C.C., 2003, «The practice and politics of food system localization », Journal of rural studies, vol. 19, n 1 , pp. 33-45.

Lamine C., 2005, «Settling shared uncertainties: Local partnerships between producers and consumers », Sociologia ruralis, vol. 45, n 4 , pp. 324-245.

Lemay J.-F., 2006, « Kitsch et ambiguïtés du partenariat chez une organisation de commerce équitable en France », Economie et solidarités, vol. 37, n 2, pp. 25-41.

Le Velly R., 2006, «Le commerce équitable: des échanges marchands contre et dans le marché », Revue française de sociologie, vol. 47, n 2, pp. 319-340.

Maldidier C., 2010, «Les relations entre «Artisans du Monde » et les producteurs des pays du Sud : une éthique relationnelle est-elle envisageable ?», Cahiers Agricultures, vol. 19, $\mathrm{n}^{\circ}$ 1, pp. 5-10.

Malpass A., Cloke P., Barnett C., Clarke N., 2007, «Fairtrade urbanism? The politics of place beyond place in the Bristol Fairtrade City campaign », International Journal of Urban and Regional Research, Vol. 31, n 3, pp. 633-645.

Maréchal G. (dir.), 2008, Les circuits courts alimentaires, Dijon, Editions Educagri

Mathieu L., 2002, «Rapport au politique, dimensions cognitives et perspectives pragmatiques dans l'analyse des mouvements sociaux », Revue française de science politique, vol. $52, \mathrm{n}^{\circ} 1$, pp. 75-100.

Maystadt J.-F., 2004, « Microfinance au Nord : Un effet de mode importé du Sud ? », Mondes en développement, $\mathrm{n}^{\circ} 126$, pp. 69-82.

Morgan K., 2010, «Local and green, global and fair: the ethical foodscape and the politics of care », Environment and Planning A, vol. 42, n 8 , pp. 1852-1867.

McAdam D., Tarrow S., Tilly C., 2001, Dynamics of Contention, New York, Cambridge University Press.

Renting H., Marsden T.K., Banks J., 2003, «Understanding alternative food networks: exploring the role of short food supply chains in rural development », Environment and Planning A, vol. 35, $\mathrm{n}^{\circ}$ 3, pp. 393-411. 
Star S.L, Griesemer J., 1989, «Institutional Ecology, 'Translations' and Boundary Objects: Amateurs and Professionals in Berkeley's Museum of Vertebrate Zoology, 1907-39 », Social Studies of Science, vol. 19, n 3, pp. 387-420.

Thompson E.P., 1993, Customs in common, studies in traditional popular culture, New York, The New Press.

Tornatore J.-L., 2000, «Le patrimoine comme objet-frontière » in Fédération des Parcs Naturels Régionaux (dir.), De la connaissance à la gestion du patrimoine, Paris, Fédération des Parcs Naturels Régionaux, pp. 21-24.

Vinck D., 1999, «Les objets intermédiaires dans les réseaux de coopération scientifique. Contribution à la prise en compte des objets dans les dynamiques sociales », Revue française de sociologie, vol. 40, $\mathrm{n}^{\circ}$ 2, pp. 385-414.

Watts D.C.H., Ilbery B., Maye D., 2005, « Making reconnections in agro-food geography: alternative systems of food provision », Progress in Human Geography, vol. 29, n 1, pp. 2240. 